\title{
Teaching NeuroImages: Spindle coma following cerebral herniation and pontine infarction
}

Stefanie P. Cappucci, MD, Kyle C. Rossi, MD, and Daniel M. Goldenholz, MD, PhD

Neurology ${ }^{\circledR}$ 2020;95:e434-e435. doi:10.1212/WNL.0000000000009942

A 67-year-old woman presented with bilateral subdural hematomas. After hematoma evacuation, she was unresponsive with extensor posturing and preserved brainstem reflexes. MRI (figure 1) revealed extensive posterior circulation infarction including the mid-pons, thought secondary to herniation and vascular compression. EEG (figure 2) revealed an unreactive background resembling normal N2 sleep (including spindles, K-complexes, and vertex waves) for $>24$ hours, diagnostic of spindle coma. ${ }^{1}$ Conditions implicated in spindle coma tend to disrupt the ascending arousal system at the brainstem and hypothalamic level, but spare the thalamus. ${ }^{1,2}$ Underlying etiology, extent of injury, and EEG reactivity provide more prognostic insight than spindle coma alone. ${ }^{1,2}$

Figure 1 Brain $\mathrm{MRI}$, mid pontine region, axial view

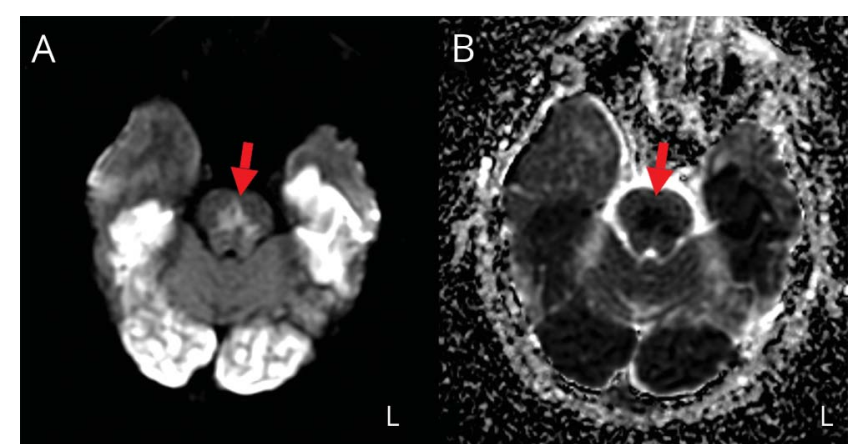

Brain MRI demonstrates extensive acute infarction of bilateral occipital lobes, bilateral posterior temporal lobes, and mid pons (arrows) on diffusion -weighed imaging (A) and adjusted diffuse coefficient imaging (B).

\section{Study funding}

No targeted funding reported.

\section{Disclosure}

The authors report no disclosures relevant to the manuscript. Go to Neurology.org/ $\mathrm{N}$ for full disclosures.

\author{
Correspondence \\ Dr. Cappucci \\ spcappuc@ \\ bidmc.harvard.edu
}

\section{MORE ONLINE}

$\rightarrow$ Teaching slides

links.lww.com/WNL/

B133 


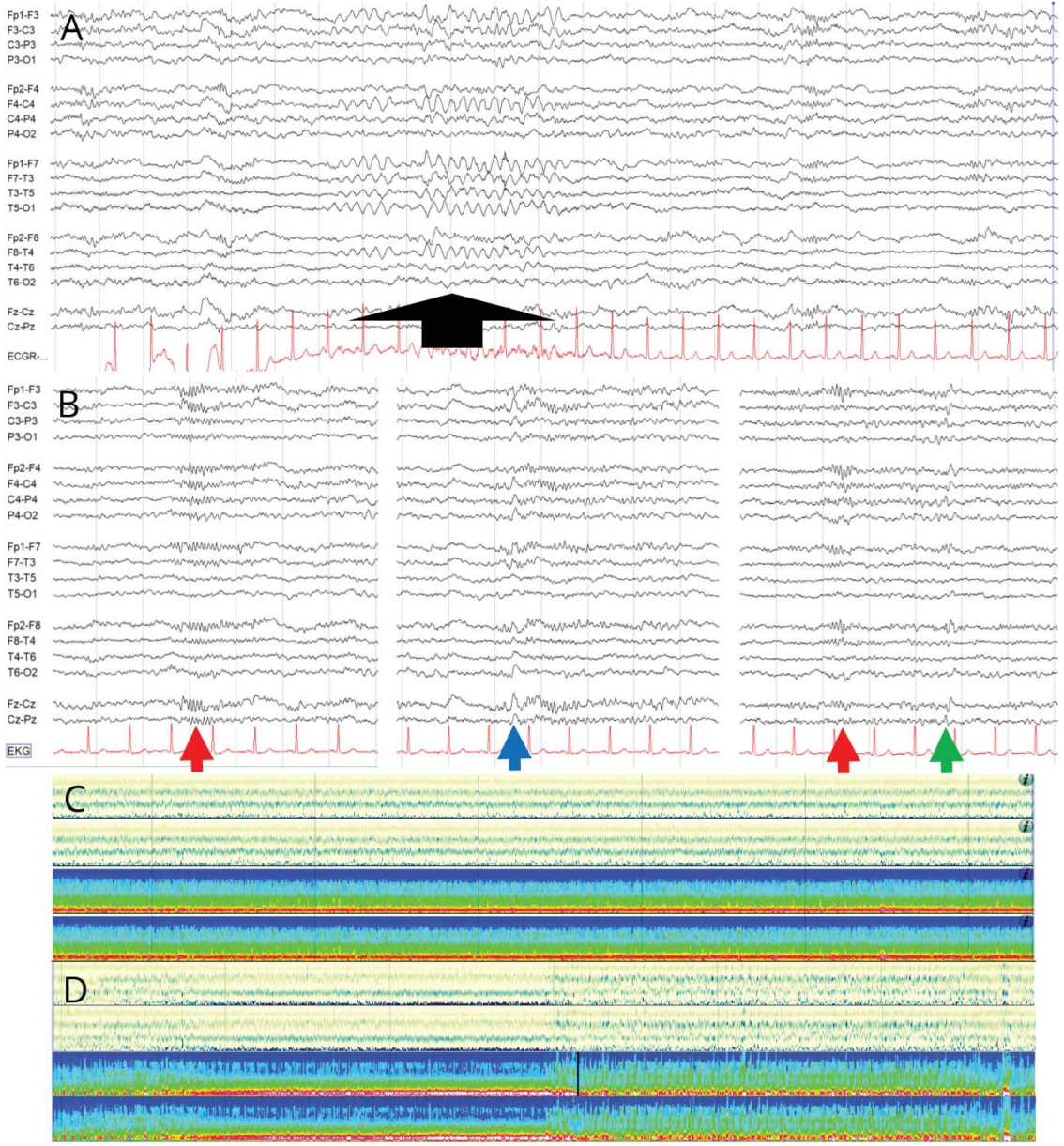

EEG (A) in longitudinal bipolar montage is without change after sternal rub (arrow), but normal N2 sleep graphoelements (B) are demonstrated: sleep spindles (red arrows), K-complex (blue arrow), and vertex wave (green arrow). Two hours of quantitative EEG rhythmicity and power spectrogram (C) do not show the frequency variability of normal sleep cycling as seen in a typical noncomatose patient (D).

\section{Appendix Authors}

\begin{tabular}{lll}
\hline Name & Location & Contribution \\
\hline $\begin{array}{l}\text { Stefanie P. } \\
\text { Cappucci, MD }\end{array}$ & $\begin{array}{l}\text { Beth Israel Deaconess } \\
\text { Medical Center, } \\
\text { Boston, MA }\end{array}$ & $\begin{array}{l}\text { Designed and conceptualized } \\
\text { case report, drafted and } \\
\text { revised manuscript }\end{array}$ \\
\hline $\begin{array}{l}\text { Kyle C. Rossi, } \\
\text { MD }\end{array}$ & $\begin{array}{l}\text { Beth Israel Deaconess } \\
\text { Medical Center, } \\
\text { Boston, MA }\end{array}$ & $\begin{array}{l}\text { Designed and conceptualized } \\
\text { case report, revised } \\
\text { manuscript }\end{array}$ \\
\hline $\begin{array}{l}\text { Daniel M. } \\
\text { Goldenholz, } \\
\text { MD, PhD }\end{array}$ & $\begin{array}{l}\text { Beth Israel Deaconess } \\
\text { Medical Center, } \\
\text { Boston, MA }\end{array}$ & $\begin{array}{l}\text { Revised and edited the } \\
\text { manuscript }\end{array}$ \\
\hline
\end{tabular}

\section{References}

1. Britt CW Jr. Nontraumatic "spindle coma": clinical, EEG and prognostic features. Neurology 1981;31:393-397.

2. Seet RCS, Lim ECH, Wilder-Smith EPV. Spindle coma from acute midbrain in farction. Neurology 2005;64:2159-2160. 


\section{Neurology}

\section{Teaching NeuroImages: Spindle coma following cerebral herniation and pontine infarction}

Stefanie P. Cappucci, Kyle C. Rossi and Daniel M. Goldenholz

Neurology 2020;95;e434-e435 Published Online before print June 29, 2020

DOI 10.1212/WNL.0000000000009942

This information is current as of June 29, 2020

\section{Updated Information \& Services}

References

Subspecialty Collections

Permissions \& Licensing

Reprints including high resolution figures, can be found at: http://n.neurology.org/content/95/4/e434.full

This article cites 2 articles, 2 of which you can access for free at: http://n.neurology.org/content/95/4/e434.full\#ref-list-1

This article, along with others on similar topics, appears in the following collection(s):

\section{Coma}

http://n.neurology.org/cgi/collection/coma

EEG

http://n.neurology.org/cgi/collection/eeg_

\section{Infarction}

http://n.neurology.org/cgi/collection/infarction

Information about reproducing this article in parts (figures,tables) or in its entirety can be found online at:

http://www.neurology.org/about/about_the_journal\#permissions

Information about ordering reprints can be found online:

http://n.neurology.org/subscribers/advertise

Neurology ${ }^{\circledR}$ is the official journal of the American Academy of Neurology. Published continuously since 1951, it is now a weekly with 48 issues per year. Copyright () 2020 American Academy of Neurology. All rights reserved. Print ISSN: 0028-3878. Online ISSN: 1526-632X.

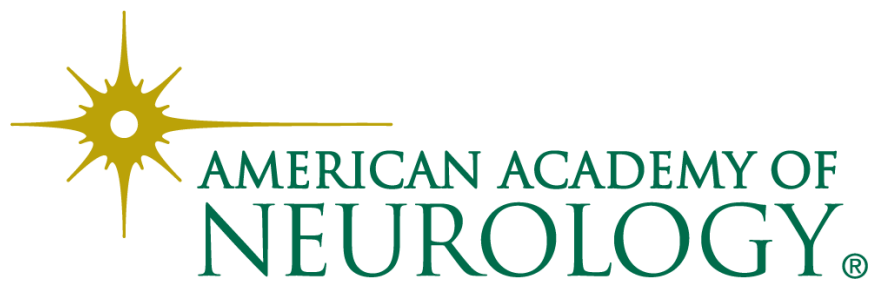

